\title{
Impacto das mudanças climáticas sobre a leishmaniose no Brasil
}

\author{
The impact of climate change on leishmaniasis in Brazil
}

Chrystian Soares Mendes ${ }^{1}$

Alexandre Bragança Coelho ${ }^{2}$

José Gustavo Féres ${ }^{3}$

Elvanio Costa de Souza ${ }^{2}$

Dênis Antônio da Cunha²

${ }^{1}$ Instituto de Ciências Sociais Aplicadas, Universidade Federal de Ouro Preto. R. do Catete 166, Centro. 35420-000 Mariana MG Brasil. chrystiansm@hotmail.com ${ }^{2}$ Departamento de Economia Rural, Centro de Ciências Agrárias, Universidade Federal de Viçosa.

${ }^{3}$ Instituto de Pesquisa Econômica Aplicada.
Abstract This paper sought to assess how climate change will affect the proliferation of leishmaniasis in Brazil in three time frames: 2010-2039, 2040-2079 and 2080-2100, and with two climate change scenarios. The relation of temperature, precipitation and the number of hospital admissions due to leishmaniasis was estimated and projections were made using these results. Results show that precipitation has a strong relation with leishmaniasis incidence and projections show that by the end of the twenty-first century there will be a $15 \%$ growth in the annual number of hospital admissions due to leishmaniasis in Brazil, compared to the base scenario (1992-2002). In regional terms, projections indicate growth in every region, with the exception of the Mid-West. The highest relative growth will be in the South of the country, while the highest increase in absolute terms will be observed in the Northeast region. In general, the incidence of leishmaniasis will grow in Brazil due to climate change.

Key words Climate change, Health, Leishmaniasis, Brazil
Resumo Este estudo buscou verificar como as mudanças climáticas podem afetar a proliferação das leishmanioses no Brasil, em três períodos, 2010-2039, 2040-2079 e 2080-2100 e dois cenários de mudanças climáticas. Realizou-se uma estimação da relação entre temperatura, precipitação e números de internações por leishmaniose e, posteriormente, a equação estimada foi utilizada para prever o impacto da mudança climática na proliferação da doença no Brasil até o fim do século XXI. Os resultados encontrados indicam que a precipitação possui forte relação com a incidência de leishmaniose e as projeções indicam que haverá uma elevação, para o final do século, da quantidade anual de internações por essa doença, em cerca de $15 \%$, em relação a 1992-2002 (cenário base). Em termos regionais, as projeções indicam crescimento em todas as regiões, com exceção do Centro-Oeste. No Sul do país haverá o maior crescimento relativo no número de internações anuais, ao passo que no Nordeste haverá o maior aumento absoluto. No geral, verifica-se que a leishmaniose aumentará sua incidência no país com a mudança climática.

Palavras-chave Mudança climática, Saúde, Leishmaniose, Brasil 


\section{Introdução}

Questões relacionadas às mudanças climáticas têm sido cada vez mais debatidas e estudadas por cientistas das mais diversas áreas do conhecimento (International Panel on Climate Change - IPCC $)^{1}$. A importância que vem sendo dada ao fenômeno é explicada pelas incertezas que o envolvem e suas consequências ainda não totalmente conhecidas. É esperado que as mudanças do clima tenham efeito em várias esferas sociais inter-relacionados (por exemplo, agricultura, silvicultura, saúde, energia, economia, migração etc.), o que aumenta sua complexidade (Food and Agriculture Organization of the United Nations - FAO $)^{2}$.

Prevê-se que a saúde humana será uma das áreas que mais sofrerão com os efeitos das mudanças climáticas ${ }^{3}$. Barcellos et al. ${ }^{4}$ destacam que tais efeitos podem ser diretos, como as doenças e mortes causadas por ondas de calor e eventos extremos, ou indiretos, tais como aqueles provocados por modificação do ecossistema, dos ciclos biogeoquímicos que permitem a elevação de doenças infecciosas e também daquelas não transmissíveis, como desnutrição.

Em relação aos efeitos indiretos, cabe destacar o papel das alterações climáticas sobre a transmissão de doenças vetoriais. Em alguns casos, a seca muito prolongada reduz a atividade do mosquito transmissor (vetor), isto é, reduz seu tempo de vida e dificulta sua procriação, ao passo que aumenta o número de pessoas não imunes, pois as ondas de calor reduzem a imunidade da pele de parte da população, tornando-as mais suscetíveis ao contágio por vírus, fungos etc., o que permite o aumento da proliferação de doenças transmitidas por mosquitos (dengue, leishmanioses, malária, febre amarela) $)^{3}$. Ao contrário das secas, conforme Kunh et al. ${ }^{5}$, as chuvas têm efeito indireto sobre a longevidade dos vetores, pois grande parte do desenvolvimento de alguns mosquitos transmissores se dá através da água parada. Esses fatores, em correlação com a falta de saneamento básico, elevam a distribuição geográfica e a abundância sazonal de doenças vetoriais.

Uma dessas doenças vetoriais que merecem destaque, principalmente no caso de países em desenvolvimento como o Brasil, é a leishmaniose. Estima-se que cerca de 350 milhões de pessoas no mundo corram risco de serem infectadas por essa doença, sendo que 12 milhões já estão e aproximadamente dois milhões de novos casos ocorram a cada $a n o^{6}$.
Há evidências da existência de uma relação entre as condições climáticas e a transmissão de doenças vetoriais como dengue e leishmanio$\mathrm{se}^{7-11}$. Segundo Rey ${ }^{12}$, há indícios de que o período de maior transmissão da leishmaniose ocorra durante e logo após a estação chuvosa, quando há um aumento da densidade populacional do inseto nos ambientes urbanos e nas periferias de grandes centros. O Departamento de Vigilância Epidemiológica do Ministério da Saúde do Brasil $^{13}$ afirma que o mosquito da leishmaniose adapta-se facilmente ao peridomicílio e às variadas temperaturas, o que permite que seja encontrado no interior dos domicílios e abrigos de animais domésticos.

As mudanças climáticas podem afetar a incidência das leishmanioses tanto no Brasil como no mundo. Entretanto, são raros estudos como o de Franke et al. ${ }^{10}$, que analisam a relação entre padrões climáticos e incidência da leishmaniose no Brasil. A maioria dos estudos existentes aborda apenas o impacto que a doença possui sobre a saúde humana, a elevação do número de casos da doença, mas não sua relação com as mudanças climáticas, o que permite a este estudo contribuir de forma relevante para a literatura. Previsões podem ser úteis para que o governo e a sociedade adotem medidas de combate à doença após eventos que favorecem sua proliferação e contaminação (chuvas intensas, seguidas de períodos secos). Ao antecipar estes eventos, o estado pode desenvolver políticas públicas para proteger a população; criar campanhas de sensibilização e conscientização mais efetivas com intuito de reduzir o número de casos e os elevados custos para o tratamento da zoonose.

De forma resumida, o problema que se coloca é entender e quantificar a relação entre precipitação, temperatura e proliferação de leishmaniose no Brasil e, em particular, avaliar como a mudança climática prevista para este século irá afetar a disseminação dessa doença no país. Sendo assim, este trabalho tenta responder às seguintes perguntas: As mudanças climáticas podem afetar a proliferação da leishmaniose no Brasil? Quais regiões do país seriam as mais afetadas? Dessa forma, o objetivo é verificar como a temperatura e a precipitação podem afetar a disseminação das leishmanioses nas regiões e unidades da federação do Brasil, bem como projetar a variação do número de internações das leishmanioses em três períodos, 2010-2039, 2040-2079 e 2080-2100, em dois cenários de mudanças climáticas. 


\section{Metodologia}

Para alcançar os objetivos apresentados, o presente estudo baseou-se nos de Deschênes e Greenstone ${ }^{14}$ e Souza et al. ${ }^{15}$, que analisaram o efeito da temperatura sobre a taxa de mortalidade nos Estados Unidos e no Brasil, respectivamente. Mas, diferentemente, este trabalho procura captar o efeito da precipitação e da temperatura sobre a proliferação da leishmaniose no Brasil. Em outras palavras, o foco foi sobre a morbidade e não sobre a mortalidade.

Sendo assim, a seguinte equação foi ajustada para o número de internações por leishmanioses:

$Y_{i t}=\sum_{j} \theta_{j}^{T M E D} T M E D_{i t j}+\sum_{l} \delta_{l}^{\text {PREC }} \operatorname{PREC}_{i t l}{ }^{+} \alpha_{i+} \gamma_{s t=} \varepsilon_{i t}$

em que $Y_{i t}$ é o número de internações hospitalares por leishmaniose, no município $i$ no ano t. A variável $T M E D_{i t j}$ representa o número de meses, no ano $t$ e no município $i$, em que a temperatura média mensal esteve em uma das cinco $j$ faixas (abaixo de 20, 20-23, 23-26, 26-29, e acima de $29^{\circ} \mathrm{C}$ ), tais faixas correspondem ao intervalo em que os mosquitos transmissores podem se desenvolver ou não, conforme relata Rey ${ }^{12}$. De acordo com Souza et al. ${ }^{15}$, o emprego desta estratégia permite captar as relações não lineares entre a temperatura e a variável dependente (neste estudo, o número de internações por leishmaniose).

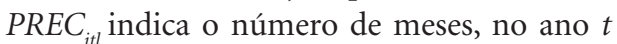
e no município $i$, em que a precipitação mensal esteve em uma das cinco $l$ faixas (abaixo de 10, 10-50, 50-100, 100-150, e acima de $150 \mathrm{~mm}$ ); $\alpha_{i}$ são um conjunto de efeitos fixos de município, que captam os determinantes do número de internações por leishmaniose que são invariantes ao longo do tempo, específicos de cada município e não observados. Como destacaram Souza et al. ${ }^{15}$, o uso desses efeitos fixos visa a evitar que as diferenças na qualidade hospitalar permanente ou na saúde geral da população local perturbem os efeitos das variáveis climáticas sobre a variável dependente. $\gamma_{s t}$ são efeitos fixos de estado, por ano, que controlam as mudanças temporais na variável dependente que são comuns em determinado estado (como por exemplo, alterações nas políticas de saúde estadual); e $\varepsilon_{i t}$ é o termo de erro estocástico.

Como destacado, nos períodos quentes, após as estações chuvosas, há uma tendência de aumento da densidade de mosquitos transmissores da leishmaniose. Por isso, na equação (1) se utiliza a distribuição das temperaturas médias mensais durante o ano, sendo que os resultados de sua estimação devem ser livres dos impactos defasados. Isso é feito, pois o efeito de meses chuvosos e quentes sobre a proliferação da doença pode levar algum tempo para ser sentido. Segundo Rey ${ }^{12}$, o número de casos de pacientes infectados por leishmanioses começa a aumentar cerca de 90 dias após os períodos de chuva. Como foram utilizados dados anuais de morbidade, as estimativas devem estar livres dos impactos defasados que ocorreriam em períodos menores de tempo. Com isso, os coeficientes $\theta_{j}^{T M E D}$ refletem o impacto completo de longo prazo de um mês com a temperatura média em determinada faixa.

\section{Especificação do modelo de previsão}

Realizada a estimação da relação entre temperatura, precipitação e números de internações por leishmaniose, estes dados foram utilizados para prever o impacto da mudança climática na proliferação da doença no Brasil até o fim do século XXI. Para a realização desta etapa, utilizaram-se estimativas de variações de temperatura e precipitação, baseadas nos cenários A1B e A2 do Painel Intergovernamental de Mudanças Climáticas (IPCC). Assim como em Cunha et al. ${ }^{16}$, a escolha desses cenários foi baseada em Le Quéré et al. ${ }^{17}$. Estes autores demonstram que os padrões atuais de emissão de GEE estão mais próximos aos cenários $\mathrm{A} 1 / \mathrm{A} 2$ do que aos cenários $\mathrm{B}$, os quais, por sua vez, são considerados não realistas no período atual.

De acordo com Meehl et al. ${ }^{18}$, para a o período que vai até metade do século, o cenário A1B é mais pessimista (pressupõe maior volume de emissões de gases de efeito estufa e, consequentemente, maiores impactos sobre o clima); já o cenário A2 é mais pessimista no período do final do século. Os autores estimam que a temperatura média aumentará em torno de $1,8^{\circ} \mathrm{C}$ (segundo o primeiro cenário, $\mathrm{A} 1 \mathrm{~B})$ ou $1,7^{\circ} \mathrm{C}$ (de acordo com o segundo cenário, A2). No período final do século, o cenário A2 sugere um aumento da temperatura média de aproximadamente $3,4^{\circ} \mathrm{C}$, ao passo que pelo cenário $\mathrm{A} 1 \mathrm{~B}$, este incremento será de $2,8^{\circ} \mathrm{C}$. Para a precipitação, estima-se uma elevação em ambos os cenários para o mundo como um todo.

Por meio destas informações e dos coeficientes estimados da equação (1), foi previsto o impacto das mudanças climáticas sobre o número de internações por leishmaniose nos períodos de 2010-2040, 2040-2070 e 2070-2100. Para tal, empregou-se a seguinte fórmula: 
$\operatorname{PREVINTL~}_{\mathrm{it}}=\sum_{j} \hat{\theta}_{j} \Delta T M E D_{i j}+\sum_{l} \hat{\delta}_{l} \Delta P R E C_{i l}$

em que,

PREVINTL $_{i t}$ é o número adicional de internações por leishmaniose previsto para o município $i$ no período $t$;

$\theta_{j} \delta_{l}$ são os parâmetros estimados da equação (1) para os efeitos da temperatura e da precipitação sobre o número de internações;

$\triangle T M E D_{i j}$ é diferença entre o número médio de meses por ano que o município $i$ se deparará com temperaturas na faixa $j$ no futuro (20102040, 2040-2070 e 2070-2100) e o número médio de meses por ano que ele observou temperaturas nesta mesma faixa no período 1992-2002 (o período-base)

$\triangle P R E C_{i l}$ é a diferença entre o número médio de meses por ano que o município $i$ se deparará com níveis de precipitação na faixa $j$ no futuro (2010-2040, 2040-2070 e 2070-2100) e o número médio de meses por ano em que se observou níveis de precipitação nesta mesma faixa no período 1992-2002.

\section{Fonte e tratamento dos dados}

Os dados referentes ao número de internações por leishmaniose foram obtidos junto ao Departamento de Informática do Sistema Único de Saúde - DataSUS ${ }^{19}$. Especificamente, os dados são oriundos do Sistema de Informação Hospitalar do SUS - SIH/SUS, que é gerido pelo Ministério da Saúde, por meio da Secretaria de Assistência à Saúde em conjunto com as Secretarias Estaduais e Municipais de Saúde.

A utilização do número de internações, ao invés do de casos notificados, se deu devido à falta de informações em nível municipal para estes últimos. Somente a partir de 2001 o Sistema de Informação de Agravos de Notificação (SINAN) passou a disponibilizar o número de casos por município. Antes desse período, era de responsabilidade de cada Estado da Federação enviar os dados referentes às endemias ocorridas em seus municípios para o Ministério da Saúde. Deste modo, como as informações climáticas estavam disponíveis apenas até 2002, optou-se por utilizar o número de internações nos modelos utilizados neste estudo.

Essa escolha traz, é claro, algumas implicaç̃̃es e limitações. Apenas uma parcela dos casos de leishmanioses podem ser sujeitos a internação, o que indica que as previsões de impacto deste estudo podem estar subestimadas. Além disso, se os serviços de saúde atuarem precocemente, as internações podem ser reduzidas, o que não tem nenhuma relação com as mudanças climáticas. Dessa forma, a análise dos resultados deve levar em consideração essa importante limitação da pesquisa. Entretanto, apesar de se reconhecer essa limitação, preferiu-se ainda assim utilizar o número de internações, pois a reduzida série disponível para o número de casos não permitiria captar adequadamente a variabilidade climática interanual e seus efeitos sobre a leishmaniose.

A base de dados do DataSUS apresenta uma série de informações sobre os indivíduos que contraíram a doença, como município de residência, sexo, faixa etária, mês e ano da internação, valor médio da internação, dias de permanência etc. As informações sobre morbidade por local de residência vão de 1984 a 2007, por município. Porém, somente a partir de 1992 foram disponibilizados dados para leishmaniose a nível municipal para o Brasil.

Deve-se ressaltar que até 1997, inclusive, era utilizada a 9a Revisão da Classificação Internacional de Doenças (CID-9), em que não se discriminava qual o tipo de leishmaniose (cutânea, cutânea-mucosa, visceral). A partir de 1998, passou-se a utilizar a 10a Revisão da Classificação Internacional de Doenças (CID-10), que já disponibilizava essas informações. Entretanto, apesar de já haver essa classificação em 1998, optou-se por utilizar a soma de todos os tipos de leishmaniose, pois haveria uma perda considerável de dados ao se desmembrar pelos tipos de endemias considerando-se apenas de 1998 a 2002.

Para estimar a equação (1), utilizou-se a quantidade de internações por leishmaniose para o período de 1992 a 2002 (11 anos), uma vez as informações climáticas estão disponíveis até 2002. Os dados de temperatura e precipitação observadas para o período atual foram obtidas a partir da base CL 2.0 10' do Climate Research Unit, da University of East Anglia ${ }^{20}$.

Tanto as informações sobre clima como das endemias estão em nível municipal, no entanto, alterações ocorridas no número, área e fronteira dos municípios tornam as comparações intertemporais inconsistentes. Sendo assim, para permitir estas comparações, foi preciso agrupar os municípios em áreas mínimas comparáveis (AMC), através da união ou junção das áreas modificadas e/ou criadas, ou seja, mais abrangentes ${ }^{21}$. A compatibilização das AMC foi feita pela equipe do Núcleo de Estudos e Modelos Espaciais Sistêmicos (NEMESIS), em que se considerou a base de 1970-2005.

Sendo assim, considerando-se que a base de dados utilizada, tanto para leishmaniose como 
para variáveis climáticas, é do período de 1992 a 2002 (11 anos), e que as unidades de corte seccional foram 3.652 AMC, tem-se um total 40.172 observações.

Em relação às projeções climáticas para o período de 2010-2040, 2040-2070 e 2070-2100, estas foram feitas a partir da média dos dez Modelos de Circulação Geral (GCM) do IPCC ${ }^{22}$, disponibilizados pelo Centro de Previsão de Tempo e Estudos Climáticos do Instituto Nacional de Pesquisas Espaciais (CPTEC/INPE).

\section{Resultados}

Os resultados da estimação da equação que explica o número de internações hospitalares por leishmaniose são apresentados na Tabela 1. A equação inclui, além das variáveis referentes às faixas de temperatura (TEMP) e precipitação (PREC), os efeitos fixos para municípios e estados/ano, os quais captam o impacto de variáveis não incluídas no modelo sobre as internações por leishmaniose. Muitos dos parâmetros desses efeitos fixos foram estatisticamente significativos, de modo que se não fossem incluídos, seriam obtidos resultados viesados para os coeficientes estimados de TEMP e PREC.

Pela Tabela 1, pode-se perceber uma associação entre precipitação e casos de internações por leishmaniose. A significância estatística dos coeficientes das variáveis correspondentes às faixas de maior precipitação $P R E C_{3}, P R E C_{4}$ e $P R E C_{5}$ sugere que em anos com um maior número de meses com altas taxas de precipitação observa-se um aumento das internações. Especificamente, anos com maior número de meses com níveis de precipitação acima de 50mm são os que possuem impacto positivo e significativo sobre o número de internações. Este resultado está alinhado com a literatura sobre a epidemiologia da leishmaniose, que mostra que a precipitação é fundamental para o desenvolvimento do mosquito transmissor $^{12,23}$. Deve-se observar ainda que o efeito marginal é crescente nas faixas de precipitação, sendo maior nos meses com precipitação média acima $150 \mathrm{~mm}$. Neste sentido, para um ano com maior número de meses com quantidade de chuvas nas faixas de $50-100 \mathrm{~mm}, 100-150 \mathrm{~mm}$ e acima de $150 \mathrm{~mm}$, tem-se uma elevação do número médio anual de internações, tudo o mais permanecendo constante, de $0,15,0,17$ e de 0,19 por AMC, respectivamente, em relação a faixa base de 10$50 \mathrm{~mm}$. Já a falta de significância estatística das variáveis de temperatura sugere que estas não
Tabela 1. Coeficientes estimados para o efeito da temperatura e precipitação sobre o número de internações por leishmanioses.

\begin{tabular}{lcr}
\hline \multicolumn{1}{c}{ Variáveis } & Coeficientes & Estatística t \\
\hline TEMP $_{1}$ & 0,006 & 0,400 \\
TEMP $_{3}$ & 0,032 & 0,950 \\
TEMP $_{4}$ & 0,004 & 0,080 \\
TEMP $_{5}$ & 0,041 & 0,380 \\
PREC $_{1}$ & 0,023 &, 330 \\
PREC $_{3}$ & 0,151 & $3,370^{* *}$ \\
PREC $_{4}$ & 0,170 & $3,190^{* *}$ \\
PREC $_{5}$ & 0,194 & $2,960^{* *}$ \\
Constante & $-0,470$ & $-0,840$ \\
R2 within: 0,031 & & \\
R2 between: 0,003 & & \\
R2 overall: 0,009 & & \\
F = 36,46 & & \\
\hline
\end{tabular}

Fonte: Resultados da pesquisa.

Nota

$\mathrm{TEMP}_{1}=$ número de meses, por ano, com temperatura média abaixo de $20^{\circ} \mathrm{C}$.

$\mathrm{TEMP}_{3}=$ número de meses, por ano, com temperatura média entre 23 a $26^{\circ} \mathrm{C}$.

$\mathrm{TEMP}_{4}=$ número de meses, por ano, com temperatura média entre 26 e $29^{\circ} \mathrm{C}$.

$\mathrm{TEMP}_{5}=$ número de meses, por ano, com temperatura média acima de $29^{\circ} \mathrm{C}$.

PREC $_{1}=$ número de meses, por ano, com precipitação abaixo de $10 \mathrm{~mm}$.

$\mathrm{PREC}_{3}=$ número de meses, por ano, com precipitação entre 50 e $100 \mathrm{~mm}$.

$\mathrm{PREC}_{4}=$ número de meses, por ano, com precipitação entre 100 e $150 \mathrm{~mm}$.

$\mathrm{PREC}_{5}=$ número de meses, por ano, com precipitação acima de $150 \mathrm{~mm}$.

${ }^{(*)}$ significativo a $1 \%,{ }^{(* *)}$ significativo a $5 \%,{ }^{(* *)}$ significativo a $10 \%$

As variáveis para as faixas de temperatura $20-23^{\circ} \mathrm{C}\left(\mathrm{TEMP}_{2}\right)$ e de precipitação de 10-50mm (PREC2) foram as variáveis controle da estimação.

têm impacto sobre as internações por leishmaniose.

Estudos como o de Elnaiem et al. ${ }^{24}$, que realizam um mapeamento da leishmaniose visceral e o papel das variações da chuvas na região leste do Sudão, mostram que a precipitação tem papel preponderante sobre a incidência da doença na região, ou seja, favorece sua proliferação. Os autores calcularam a correlação entre variáveis ambientais e climáticas e verificam que é forte e positiva entre a incidência da leishmaniose nas regiões do Sudão e a precipitação.

Além do trabalho de Elnaiem et al. ${ }^{24}$, outros, como os de Werneck et al. ${ }^{25}$, Silva et al. ${ }^{26}$ e Viei$\mathrm{ra}^{27}$, que analisam regiões específicas do Brasil, como Piauí, São Paulo e Minas Gerais, também 
identificam a existência dessa relação entre chuvas e incidência de leishmaniose, corroborando assim o resultado do presente estudo de que a precipitação tem efeito significativo sobre a proliferação da doença nas regiões brasileiras.

No trabalho de Werneck et al. ${ }^{25}$, os autores mostram que há uma relação positiva entre precipitação e umidade em relação ao meio ambiente, o que favorece condições propicias para a abundancia do mosquito transmissor. Já Silva et al. ${ }^{26}$ apresentam que, dada a grande variação pluviométrica nos meses de fevereiro, março e abril dos anos de 2004 e 2005, após quatro meses, o número de vetores aumentou consideravelmente.

É importante ressaltar que, apesar de as chuvas terem papel relevante na disseminação da doença, a temperatura também tem sua participação. Em conjunto, essas duas variáveis fazem elevar ainda mais a quantidade de mosquitos transmissores, isto é, não basta apenas haver chuvas se não houver uma temperatura adequada para que os vetores se desenvolvam.

Após a análise do efeito do clima no período corrente, pode-se discutir os principais resultados obtidos por meio da análise de previsão, isto é, os impactos futuros que as variáveis climáticas podem ter sobre a proliferação da leishmaniose no Brasil. Empregou-se a equação (2) para obter o número adicional de internações anuais por leishmaniose provocadas pelas mudanças climáticas para cada AMC nos períodos de 20102039, 2040-2069 e 2070-2099. Deste modo, a
Tabela 2 apresenta o resultado para a soma de todas as AMC, o que nos fornece a quantidade adicional de internações anuais em todo o Brasil. Com o objetivo de identificar o efeito individual de cada variável climática, apresentam-se os impactos isolados de temperatura e precipitação e, em seguida, o efeito conjunto dessas variáveis no número adicional de internações, bem como a variação percentual em relação ao período base (1992-2002).

As previsões mostram que para o período 2010-2039 o impacto isolado das variações na temperatura sobre o número adicional de internações anuais será pequeno; aproximadamente 0,10 no cenário $A 1 B$, o que representa uma variação de $0,002 \%$ em relação ao número médio de internações do período-base (1992-2002); já no cenário A2, espera-se uma redução de cerca de 8,7 internações por ano (queda de $0,21 \%$ relativo ao número anual médio de internações do período-base).

Entretanto, ao se analisar os outros períodos, se observa uma elevação considerável do número de internações: para o período de 2040-2069, há uma elevação de 63 e 54 internações para os dois cenários; para o final do século, verifica-se que o impacto será aproximadamente 3 vezes maior que o período anterior para o cenário $\mathrm{A} 1 \mathrm{~B}$ e de 4,5 vezes maior para o $\mathrm{A} 2$, o que representa um número adicional de internações anuais em torno de 186 e 247 para os dois cenários, respectivamente. Isso implica numa elevação, se compara-

Tabela 2. Impactos previstos das variáveis climáticas sobre o número de internações por leishmaniose.

\begin{tabular}{|c|c|c|c|c|}
\hline \multicolumn{5}{|c|}{ Período 2010-2039 } \\
\hline \multirow{2}{*}{$\begin{array}{l}\text { Variáveis } \\
\text { climáticas }\end{array}$} & \multicolumn{2}{|c|}{ Internações anuais } & \multicolumn{2}{|c|}{ Variação no número de internações (\%) } \\
\hline & A1B & A2 & A1B & A2 \\
\hline Temperatura & 0,10 & $-8,72$ & 0,002 & $-0,211$ \\
\hline Precipitação & 383,19 & 375,10 & 9,284 & 9,083 \\
\hline Temperatura e precipitação & 383,29 & 366,38 & 9,286 & 8,877 \\
\hline \multicolumn{5}{|c|}{ Período 2040-2069 } \\
\hline Temperatura & 63,49 & 54,79 & 1,537 & 1,327 \\
\hline Precipitação & 383,98 & 377,74 & 9,301 & 9,150 \\
\hline Temperatura e precipitação & 447,47 & 432,53 & 10,841 & 10,479 \\
\hline \multicolumn{5}{|c|}{ Período 2070-2099 } \\
\hline Temperatura & 186,10 & 246,85 & 4,507 & 5,978 \\
\hline Precipitação & 376,89 & 371,21 & 9,129 & 8,991 \\
\hline Temperatura e precipitação & 562,99 & 618,06 & 13,640 & 14,974 \\
\hline
\end{tabular}

Fonte: Resultados da pesquisa. 
da com o período base, por volta de $4 \%$ no cenário $\mathrm{A} 1 \mathrm{~B}$ e de $6 \%$ para o $\mathrm{A} 2$ até o final do século.

O efeito apenas da precipitação foi bem maior sobre o número de internações por leishmaniose do que a temperatura para todos os cenários futuros. Como se prevê um aumento do número médio de meses em faixas de precipitação maiores, acompanhado do fato de que a leishmaniose tem forte relação com esta variável, observa-se uma elevação considerável no número de internações. Assim, no período de 2010-2039, projeta-se um aumento nas internações anuais de aproximadamente $9 \%$ para os cenários A1B e A2, em relação ao número médio de internações no período de 1992-2002, isto é, um adicional de 383 e 375 internações por leishmaniose, respectivamente.

Em 2040-2069 e 2070-2099, a variação permanece no mesmo patamar que a previsão do período anteriormente analisado, o que indica que os efeitos não serão crescentes com o passar do tempo, devido às previsões sobre os padrões de chuvas futuras. Contudo, é importante considerar o impacto conjunto das variáveis climáticas, isto é, da temperatura e da precipitação concomitantemente. Deste modo, os efeitos futuros sobre as internações é ainda maior ao realizar este tipo de análise. No período inicial, 2010-2039, o número de internações sofre maior influência da precipitação, dada a relação negativa que a temperatura registrou. Sendo assim, houve uma quantidade adicional de internações de 383,29 e 366,38 para o cenário A1B e A2, o que corresponde a uma elevação em relação ao período base de 9 e $8 \%$, respectivamente.

Este resultado pode ser consequência do perfil dos cenários de previsão, em que, segundo Meehl et al. ${ }^{18}$ o cenário A1B é considerado mais pessimista até meados do século, ao passo que no final do século o cenário A2 se torna mais pessimista. Tal acontecimento também é verificado no presente estudo, em que até metade do século se observa um efeito maior para o A1B sobre a incidência de leishmaniose e na outra metade para o A2. Desta maneira, no período de 2040-2069, nota-se uma elevação do número de internações por leishmaniose para os dois cenários, com um aumento de aproximadamente $11 \%$ e $10 \%$ no número adicional de internações por ano em relação ao período base para os cenários A1B e A2, respectivamente, o que representa uma previsão de aumento de 447 e 432 internações. Já para o final do século (20702099), a quantidade anual adicional de internações por leishmaniose se eleva para 562 e 618 internações, sendo responsável por aumento médio de cerca de 13 e 15\% em relação a 1992-2002.
Como há uma elevada heterogeneidade entre as regiões do país em relação aos impactos esperados da mudança climática, é interessante analisar os resultados para os estados e regiões do Brasil. A Tabela 3 apresenta a previsão dos efeitos das mudanças climáticas sobre as internações por leishmaniose, por estado, para os dois cenários (A1B e A2), para o final do século (2070-2099), em termos absolutos e em relação ao número médio de internações anuais médias de 1992-2002. Os efeitos verificados para os períodos de 20102039 e 2040-2069 seguiram o mesmo padrão que o de 2070-2099, em que os impactos foram mais expressivos, isto é, passaram a se intensificar gradualmente até o final do século.

De acordo com os resultados, todas as regiões do Brasil sofrerão impactos expressivos das mudanças climáticas ao longo dos períodos observados. Como discutido anteriormente, a leishmaniose sofre uma influência maior da precipitação do que da temperatura. Deste modo, projeta-se que até o final do século haverá um crescimento drástico das internações anuais por leishmaniose, com o cenário A2 como mais pessimista (Tabela 3 ).

Os estados do Norte irão se deparar com uma elevação de até $17 \%$ em relação ao período base (1992-2002), o que equivale a um adicional de 59 internações anuais, dos quais o Pará terá maior participação. Já no Nordeste, observar-se-á um aumento de 287 internações pelo cenário A2 e de 250 para o A1B. Haverá nesses estados uma crescente concentração de meses com chuvas em faixas elevadas (como 100-150mm), o que torna favorável a proliferação dos vetores de leishmania.

No Sudeste, tem-se um incremento de cerca de 26\% em relação a 1992-2002, para os dois cenários, no número de internações por leishmaniose, o que expressa um aumento de 181 e 178 internações, para o cenário A1B e A2, respectivamente. Os estados de São Paulo e Minas Gerais serão os mais endêmicos. No Sul, aponta-se um acréscimo de aproximadamente 228 e $227 \%$ nos casos de internação para o cenário A1B e A2, respectivamente, isto é, em média mais 100 internações por ano, com os estados do Paraná e Santa Catarina apresentando as maiores participações, com cerca de $70 \%$ e $20 \%$ das internações, respectivamente.

O Centro-Oeste apresenta uma característica similar a dos outros períodos, ou seja, redução do número de casos, porém com efeitos menos intensos neste último período. No geral, essa região terá uma redução em torno de 2 e $7 \%$ se comparado com o período base. O estado do Mato Grosso do Sul é o único que apresentará uma elevação das internações, com cerca de $12 \%$ pelo 
cenário A1B e 15\% pelo A2. Goiás apresentará uma redução em torno de $46 \%$ das internações pelo cenário A1B, seguido do Mato Grosso e do Distrito Federal.

Em suma, os impactos previstos das mudanças climáticas sobre a leishmaniose tendem a ser bem elevados. Observa-se que no Sul do país haverá o maior crescimento no número de internações anuais em termos relativos, ao passo que no Nordeste haverá o maior aumento absoluto. Além disso, a região Sudeste apresentará um crescimento considerável, seguida da região Norte. O Centro-Oeste será a única região com um decréscimo do número de internações anuais ao longo dos anos. No geral, verifica-se que a leishmaniose aumentará sua incidência no país com a mudança climática.

\section{Conclusões}

Os principais resultados encontrados pelo presente estudo sugerem que a proliferação da leishmaniose possui forte relação com a precipitação, isto é, quanto maior o número de meses com chuva, maior o número de internações da

Tabela 3. Estimativa do impacto previsto das mudanças climáticas sobre a leishmaniose, segundo as Unidades da Federação considerando a variação percentual anual em relação aos casos de internação média anual de leishmaniose do período de 1992-2002, para o cenário de 2070-2099.

\begin{tabular}{|c|c|c|c|c|c|c|c|c|c|c|c|c|}
\hline \multirow[b]{3}{*}{ UF } & \multicolumn{6}{|c|}{$A 1 B$} & \multicolumn{6}{|c|}{$\mathrm{A} 2$} \\
\hline & \multicolumn{2}{|c|}{ Temp } & \multicolumn{2}{|c|}{ Precip. } & \multicolumn{2}{|c|}{ Temp + Prec } & \multicolumn{2}{|c|}{ Temp } & \multicolumn{2}{|c|}{ Precip. } & \multicolumn{2}{|c|}{ Temp + Prec } \\
\hline & Tot. ${ }^{*}$ & $(\%)$ & Tot. ${ }^{*}$ & $(\%)$ & Tot. $^{*}$ & $(\%)$ & Tot. ${ }^{*}$ & $(\%)$ & Tot. $^{*}$ & $(\%)$ & Tot. $^{*}$ & $(\%)$ \\
\hline $\mathrm{N}$ & 44.8 & 0.1 & 7.9 & 2.3 & 52.6 & 15.4 & 51.3 & 15.0 & 8.0 & 2.3 & 59.3 & 17.4 \\
\hline $\mathrm{RO}$ & 0.3 & 0.0 & 0.1 & 0.1 & 0.3 & 0.8 & 0.3 & 0.7 & 0.1 & 0.1 & 0.4 & 0.9 \\
\hline $\mathrm{AC}$ & 1.1 & 0.1 & 0.9 & 4.1 & 1.9 & 9.2 & 1.3 & 6.0 & 0.9 & 4.1 & 2.1 & 10.0 \\
\hline $\mathrm{AM}$ & 9.7 & 0.5 & 1.3 & 6.4 & 11.0 & 55.6 & 9.8 & 49.7 & 1.5 & 7.6 & 11.3 & 57.3 \\
\hline $\mathrm{RR}$ & 0.3 & 0.0 & 0.1 & 0.5 & 0.3 & 2.1 & 0.3 & 2.1 & 0.1 & 0.6 & 0.4 & 2.7 \\
\hline PA & 24.3 & 0.2 & 7.8 & 5.9 & 32.1 & 24.1 & 27.9 & 21.0 & 7.9 & 6.0 & 35.9 & 27.0 \\
\hline $\mathrm{AP}$ & 0.2 & 0.1 & 0.2 & 9.1 & 0.5 & 18.5 & 0.7 & 27.8 & 0.1 & 3.0 & 0.8 & 30.8 \\
\hline TO & 8.9 & 0.1 & -2.4 & -2.3 & 6.5 & 6.2 & 11.0 & 10.4 & -2.5 & -2.4 & 8.4 & 8.0 \\
\hline $\mathrm{NE}$ & 66.9 & 0.0 & 184.0 & 6.7 & 250.9 & 9.2 & 106.7 & 3.9 & 180.7 & 6.6 & 287.5 & 10.5 \\
\hline MA & 43.0 & 0.2 & 13.5 & 5.4 & 56.6 & 22.5 & 45.0 & 17.9 & 13.1 & 5.2 & 58.1 & 23.1 \\
\hline PI & 23.4 & 0.1 & 9.3 & 2.1 & 32.7 & 7.2 & 25.5 & 5.6 & 9.5 & 2.1 & 35.0 & 7.7 \\
\hline $\mathrm{CE}$ & 14.8 & 0.0 & 10.9 & 3.0 & 25.7 & 7.1 & 24.5 & 6.8 & 7.9 & 2.2 & 32.5 & 9.0 \\
\hline RN & 12.3 & 0.1 & 10.8 & 6.5 & 23.1 & 13.9 & 21.1 & 12.7 & 5.6 & 3.4 & 26.7 & 16.1 \\
\hline $\mathrm{PB}$ & -7.9 & -0.1 & 17.2 & 11.8 & 9.3 & 6.4 & -1.2 & -0.8 & 14.8 & 10.1 & 13.6 & 9.3 \\
\hline PE & -6.5 & -0.0 & 21.3 & 6.8 & 14.8 & 4.7 & -4.3 & -1.4 & 22.3 & 7.2 & 18.0 & 5.8 \\
\hline $\mathrm{AL}$ & -0.2 & -0.0 & 11.8 & 7.4 & 11.6 & 7.2 & 1.6 & 1.0 & 15.4 & 9.7 & 17.0 & 10.7 \\
\hline SE & 0.3 & 0.0 & 14.1 & 11.6 & 14.4 & 11.8 & 3.4 & 2.8 & 17.3 & 14.2 & 20.7 & 17.0 \\
\hline BA & -12.3 & -0.0 & 75.0 & 9.7 & 62.7 & 8.1 & -8.9 & -1.1 & 74.7 & 9.7 & 65.9 & 8.5 \\
\hline SE & 56.4 & 0.1 & 125.4 & 17.9 & 181.8 & 26.0 & 60.6 & 8.7 & 117.7 & 16.8 & 178.3 & 25.5 \\
\hline MG & 36.9 & 0.1 & 8.0 & 2.4 & 44.9 & 13.5 & 28.4 & 8.6 & 15.9 & 4.8 & 44.3 & 13.4 \\
\hline ES & 2.5 & 0.1 & 0.3 & 1.2 & 2.8 & 9.8 & 3.1 & 11.0 & 0.7 & 2.5 & 3.8 & 13.4 \\
\hline RJ & 2.2 & 0.0 & 3.0 & 3.5 & 5.3 & 6.1 & 3.0 & 3.5 & 2.7 & 3.1 & 5.7 & 6.6 \\
\hline SP & 14.8 & 0.1 & 114.0 & 45.1 & 128.8 & 51.0 & 26.1 & 10.3 & 98.4 & 39.0 & 124.5 & 49.3 \\
\hline$S$ & 17.5 & 0.4 & 83.6 & 188.8 & 101.1 & 228.3 & 16.4 & 37.1 & 84.0 & 189.8 & 100.5 & 226.9 \\
\hline PR & 13.8 & 0.4 & 59.2 & 155.3 & 73.0 & 191.6 & 11.6 & 30.5 & 58.6 & 153.8 & 70.2 & 184.3 \\
\hline SC & 5.2 & 1.3 & 12.3 & 301.1 & 17.5 & 428.5 & 6.8 & 167.1 & 12.6 & 309.0 & 19.5 & 476.0 \\
\hline RS & -1.5 & -0.7 & 12.1 & 580.2 & 10.6 & 506.8 & -2.0 & -96.6 & 12.8 & 612.6 & 10.8 & 515.9 \\
\hline $\mathrm{CO}$ & 0.5 & 0.0 & -23.9 & -7.9 & -23.4 & -7.8 & 11.7 & 3.9 & -19.2 & -6.4 & -7.5 & -2.5 \\
\hline MS & 4.0 & 0.1 & 5.7 & 7.2 & 9.6 & 12.3 & 5.7 & 7.2 & 6.0 & 7.6 & 11.7 & 14.9 \\
\hline MT & 3.4 & 0.1 & -4.8 & -8.8 & -1.4 & -2.5 & 4.3 & 7.9 & -4.8 & -8.9 & -0.5 & -1.0 \\
\hline $\mathrm{GO}$ & -6.8 & -0.1 & -24.8 & -36.5 & -31.6 & -46.5 & 1.9 & 2.7 & -20.3 & -30.0 & -18.5 & -27.2 \\
\hline DF & -0.1 & -0.0 & -0.0 & -0.0 & -0.1 & -0.1 & -0.1 & -0.1 & -0.0 & -0.0 & -0.1 & -0.1 \\
\hline
\end{tabular}

Fonte: Resultados da pesquisa.

${ }^{(*}$ : Total previsto de internações por leishmaniose. 
doença em meses subsequentes. As estimativas da quantidade adicional de casos de internação por leishmaniose nos períodos de 2010-2039, 20402069 e 2070-2099 indicaram que haverá uma forte elevação em quase todas as regiões do país. A previsão para o último período é de uma elevação de aproximadamente 618 e 562 casos adicionais anuais de internação pela endemia no país, pelos cenários A2 e A1B, respectivamente, o que equivale a um aumento de cerca de $15 \%$ e $13 \%$ em relação ao período base. Estados como Rondônia, Pará, Amazonas, Bahia, Maranhão, Minas Gerais, São Paulo, Santa Catarina e Mato Grosso do Sul serão os mais afetados pela leishmaniose em virtude das alterações climáticas.
Esses resultados indicam aos governos $(\mathrm{Mu}-$ nicipal, Estadual e Federal) dois caminhos possíveis: mitigação ou adaptação. O primeiro seria de combate à proliferação da doença nas regiões que devem ser mais afetadas, tentando diminuir o número futuro de casos. Politicas como aumento da rede de saneamento básico, combate aos focos do mosquito transmissor etc. podem diminuir o número de casos esperados com a mudança climática. O segundo caminho seria a preparação dos sistemas públicos de saúde para a recepção e tratamento dos casos adicionais de leishmaniose. Previsões detalhadas como as fornecidas por este estudo podem ser úteis no planejamento desta estratégia.

\section{Colaboradores}

CS Mendes trabalhou na concepção, delineamento, redação final e interpretação dos resultados; $\mathrm{AB}$ Coelho na revisão crítica, delineamento e aprovação da versão a ser publicada; JG Féres na análise, interpretação dos dados e revisão crítica; EC Souza na metodologia de pesquisa e interpretação dos resultados; e DA Cunha, na pesquisa e na metodologia.

\section{Agradecimentos}

Agradecemos a um parecerista da Revista pelas considerações sobre as limitações do uso do número de internações como medida de impacto. 
Referências

1. International Panel on Climate Change (IPCC). Climate Change 2013: The Physical Science Basis. Geneva: IPCC; 2013

2. Food and Agriculture Organization of the United Nations (FAO). Facing the challenges of climate change and food security: The role of research, extension and communication for development. FAO; 2013. [acessado $2013 \mathrm{fev}$ 15]. Disponível em: http://www.fao.org/docrep/018/ i3334e/i3334e.pdf

3. Confalonieri, U, Menne B, Akhtar R, Ebi KL, Hauengue M, Kovats RS, Revich B, Woodward A. Human health. In: Parry ML, Canziani OF, Palutikof JP, Van Der Linden PJ, Hanson CE, editors. Climate change 2007: Impacts, Adaptation and Vulnerability. Contribution of Working Group II to the Fourth Assessment Report of the Intergovernmental Panel on Climate Change. Cambridge: Cambridge University Press; 2007. p. 391-431.

4. Barcellos C, Monteiro AMV, Corvalán C, Gurgel H, Carvalho MS, Artoxo P, Hacon S, Ragoni V. Climatic and environmental changes and their effect on infectious diseases: scenarios and uncertainties for Brazil. Epidemiologia e Serviços de Saúde 2009; 18(3):285-304.

5. Kuhn K, Campbell-Lendrum D, Haines A, Cox J. Using climate to predict infectious disease epidemics. Geneva: World Health Organization; 2005.

6. World Health Organization (WHO). Department of control of negletecd tropical diseases (NTD). Neglected tropical diseases, hidden successes, emerging opportunities. Geneva: WHO; 2009.

7. Beebe NW, Cooper RD, Mottram P, Sweeney AW. Aus tralia's dengue risk driven by human adaptation to climate change. PLoS Negl Trop Dis 2009; 3(5):e429.

8. Reiter P. Climate and mosquito-borne disease. Environ Health Perspect 2001; 109(Supl. 1):141-161.

9. Tseng WC, Chen CC, Chang CC, Chu YH. Estimating the economic impacts of climate change on infectious diseases: a case study on dengue fever in Taiwan. Climatic Change 2009; 92(1-2):123-140.

10. Franke CR, Ziller M, Staubach C, Latif M. Impact of the El Niño/Southern oscillation on visceral leishmaniasis, Brazil. Emerg Infect Dis 2002; 8(9):914-917.

11. Sharma U, Singh S. Insect vectors of Leishmania: distribution, physiology and their control. J. Vector Borne Dis 2008; 45(4):255-272.

12. Rey L. Parasitologia: parasitos e doenças parasitárias do homem nos trópicos ocidentais. $4^{\text {a }}$ ed. Rio de Janeiro: Guanabara Koogan; 2008. p.359-412.

13. Brasil. Ministério da Saúde (MS). Secretária de Vigilância em Saúde. Departamento de Vigilância Epidemiológica. Manual de vigilância e controle da leishmaniose visceral. Brasília: Editora do Ministério da Saúde; 2003.

14. Deschênes O, Greenstone M. Climate change, mortality, and adaptation: evidence from annual fluctuations in weather in the US. NBER Working Paper Series, n. 13178. Cambridge (USA): National Bureau of Economic Research, jun. 2007. [2011 abr 5]. Disponível em: http://www.nber.org/papers/w13178

15. Souza ES, Coelho AB, Lima JE, Cunha DA, Féres JG. Impacto das mudanças climáticas sobre o bem-estar relacionado à saúde no Brasil. Pesquisa e Planejamento Econômico - PPE 2013; 43(1):49-87.
16. Cunha DA, Coelho AB, Féres JG, Braga MJ. Irrigação como estratégia de adaptação de pequenos agricultores às mudanças climáticas: aspectos econômicos. Rev Economia e Sociologia Rural 2013; 51(2):369-386.

17. Le Quéré C, Raupach MR, Canadell JG, Marland G. Trends in the sources and sinks of carbon dioxide. $\mathrm{Na}$ ture Geoscience 2009; 689:1-6.

18. Meehl GA, Stocker TF, Collins WD, Friedlingstein P, Gaye AT, Gregory JM, Kitoh A, Knutti R, Murphy JM, Noda A, Raper SCB, Watterson IG, Weaver AJ, Zhao ZC. Global climate projections. In.: Solomon S, Qin D, Manning M, Chen Z, Marquis M, Averyt KB, Tignor M, Miller HL. Climate change 2007: the physical science basis. Cambridge: Cambridge University Press; 2007. p. 747-846.

19. Sistema de Informações sobre Mortalidade/Sistema Único de Saúde (SUS). Base de dados do departamento de informática do Sus - Datasus. [acessado 2011 jun 18]. Disponível em: http://www.datasus.gov.br

20. Climatic Research Unit (CRU). High-resolution gridded datasets. Norwich: CRU. [acessado 2012 jun 27]. Disponível em: http://www.cru.uea.ac.uk/

21. Reis E, Pimentel M, Alvarenga AI. Áreas mínimas comparáveis para os períodos intercensitários de 1872 a 2000. 2007. [acessado 2011 set 11]. Disponível em: http:// www.ipeadata.gov.br/doc/AMC-1872-2000.doc

22. International Panel on Climate Change (IPCC). Cli mate change 2007: Synthesis Report. Contribution of Working Groups I, II and III to the Fourth Assessment Report of the Intergovernmental Panel on Climate Change. Geneva: IPCC; 2007.

23. Brasil. Ministério da Saúde (MS). Secretária de Vigilância em Saúde. Departamento de Vigilância Epidemiológica. Guia de vigilância epidemiológica. Brasília: Editora do Ministério da Saúde; 2008.

24. Elnaiem D-EA, Schorscher J, Bendall A, Obsomer V, Osman ME, Mekkawi AM, Connor SJ, Ashford RW Thomson MC. Risk mapping of visceral leishmaniasis: The role of local variation in rainfall and altitude on the presence and incidence of kala-azar in eastern Sudan. Am J Trop Med Hyg 2003; 68(1):10-17.

25. Werneck GL, Costa CHN, Walker AM, David JR, Wand M, Maguire JH. Multilevel modeling of the incidence of visceral leishmaniasis in Teresina, Brazil. Epidemiol Infect 2007; 135(2):195-201.

26. Silva JGD, Wernerck GL, Cruz MSP, Costa CHN, Mendonça IL. Infecção natural de Lutzomyia longipalpis por Leishmania sp. em Teresina, Piauí, Brasil. Cad Saude Publica 2007; 23(7):1715-1720.

27. Vieira GSS. Fauna de Aedes (Ochlerotatus) Scapularis e as alterações ambientais provocadas pela construção das barragens das usinas hidrelétricas capim branco e capim branco no rio Araguari, no município de Uberlândia, Minas Gerais - Brasil [dissertação]. Uberlândia: Universidade Federal de Uberlândia; 2007.

Artigo apresentado em 23/03/2015

Aprovado em 22/04/2015

Versão final apresentada em 24/04/2015 